\title{
THE POSSIBILITIES OF HIGHER FLEXIBILITY THROUGH NEW EMPOWERED ENERGY COMMUNITIES
}

\author{
Manuel Serrano Matoses ${ }^{1}$, Lola Alacreu García ${ }^{2}$, Álvaro Nofuentes Prieto ${ }^{3}$ \\ ${ }^{1,2} 3$ Department of Technology. ETRA I+D, Valencia, Spain \\ technology-projects.etraid@grupoetra.com
}

Keywords: flexibility; empowerment; aggregation; energy community.

\begin{abstract}
Distributed Energy Resources (DER) facilitate the empowerment of consumers, as well as the emergence of new flexibility services. Prosumers need to have a higher control over their production and consumption capabilities, and this can only be achieved through a direct control over the data being captured by a next generation of smart meters enabling the owner of the data to trade with it and access added value services by aggregators. Taking control over the ownership of energy data, leads to new opportunities for energy communities. Energy communities will not on ly be able to trade their aggregated production, but also their aggregated consumption and storage flexibility.
\end{abstract}

\section{Introduction}

The increasing share of Renewable Energy Sources (RES) and storage units connected to the distribution grid has become key to improve the carbon footprint of the European electricity system and achieve energy and climate change policy goals.

Distributed Energy Resources (DER) facilitate the empowerment of consumers, as well as the emergence of new services and the thriving of new players such as energy communities and aggregators which add value to the services being traded. Key factors to ensure the success and take up of these energy communities and businesses opportunities are openness, transparency, empowerment of consumers/citizens and the provision of tools and conditions which facilitate direct citizen engagement and fair competition in the market.

Prosumers need to have a higher control over their production and consumption capabilities - i.e. flexibility, and this can only be achieved through a direct control over the data being captured by specific monitoring devices, a next generation of smart meters enabling the owner of the data to trade with it and access added value services provided not by the System Operator, but by a professional third party: an aggregator.

This first step, taking control over the ownership of energy data, leads to new opportunities for energy communities, not only in terms of fairer tariffs, but also as a relevant peer in the energy markets. Energy communities will not only be able to trade their aggregated production, but also their aggregated consumption and storage flexibility.

Moreover, the high penetration of DER in the distribution grid will make necessary a smoother cooperation between
Distribution and Transmission System Operators (DSO\&TSO).

his document, saved in the "Word 97-2003" format, is a guide to using the Manuscript Template. Before submitting your final paper, check that the format conforms to this guide. In particular, check the text to make ensure that the correct referencing style has been used and that the citations are in numerical order throughout the text. Your manuscript cannot be accepted for publication unless all formatting requirements are met.

\section{Control your data: next generation smart meters}

In the context of the H2020 Project NOBEL GRID [1], it has been designed and developed from scratch a next generation Smart Meter (SLAM) that enables the direct control of energy data flows by end-users.

Smart meters are the most obliquus equipment in a low voltage network. This is also the network level where most of the smart grid developments will have important impact. Millions of smart meters have been already deployed in LV networks, however most of them do not cover yet functionalities to leverage the smart grid potential, neither the potential for new dynamic markets of energy and especially of energy services.

In this context, the SLAM is an advanced multi-function digital single-phase smart meter Class $B$ in active energy and Class 2 in reactive. The SLAM has been certified and complies with the European legislation related to energy meters (MID) EN 50470-1 and EN 50470-3 whereby approves the installation of the meter in any country of the European Union. 
Thanks to the Linux based module at the core of the SLAM, it is possible to deploy new advanced features during the entire lifetime of the smart meter, considering in this way the evolution of the Smart Grid where it is connected. Moreover, this module adds the capability to interface with different actors in the Energy Market, not only DSOs, but also other professional third parties as aggregators.

The SLAM is based on the smart meter Unbundled concept, differentiating two sections: the metrological (SMM: Smart Metrological Meter) and the intelligence (SMX: Smart Extended Meter).

- The SMM is the part of the SLAM on the right side of the picture, which involves the metrology functionalities, as the traditional digital meters -i.e. power control and billing.

- The SMX is the part of the SLAM on the left side of the picture, which involves additional functionalities, not included in the traditional digital meters -i.e. measurement of the quality of supply and control of intelligent devices.

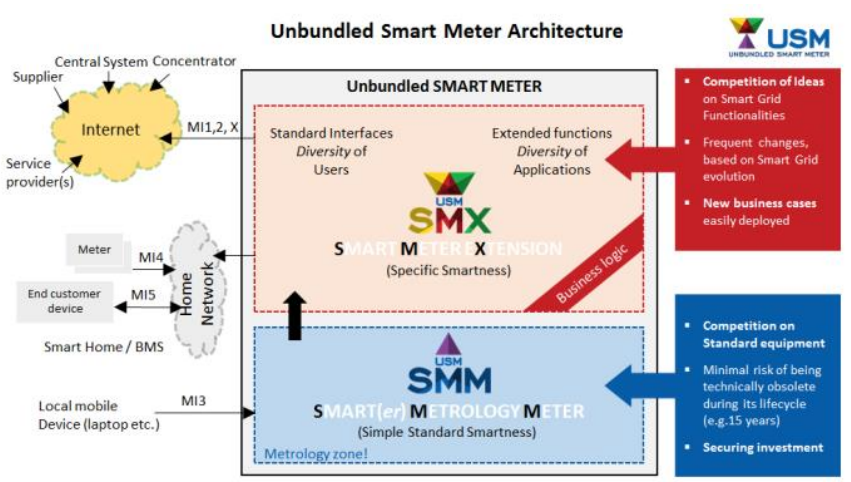

Fig. 1. Unbundled Smart Meter architecture

Hence, thanks to this USM architecture, the smart meter becomes a complex device that can cover multiple purposes in order to provide benefits for all the actors of intelligent networks.

For instance, in the case of the Operators of the distribution grid (DSO), the SLAM provides real-time information to control quality and security of the power supply. The SLAM enables the real time monitoring and management of the flexibility of the grid, giving higher control of the distributed production and consumption capabilities of the energy network. These services makes possible the maintenance and easier operation of the distribution grid.
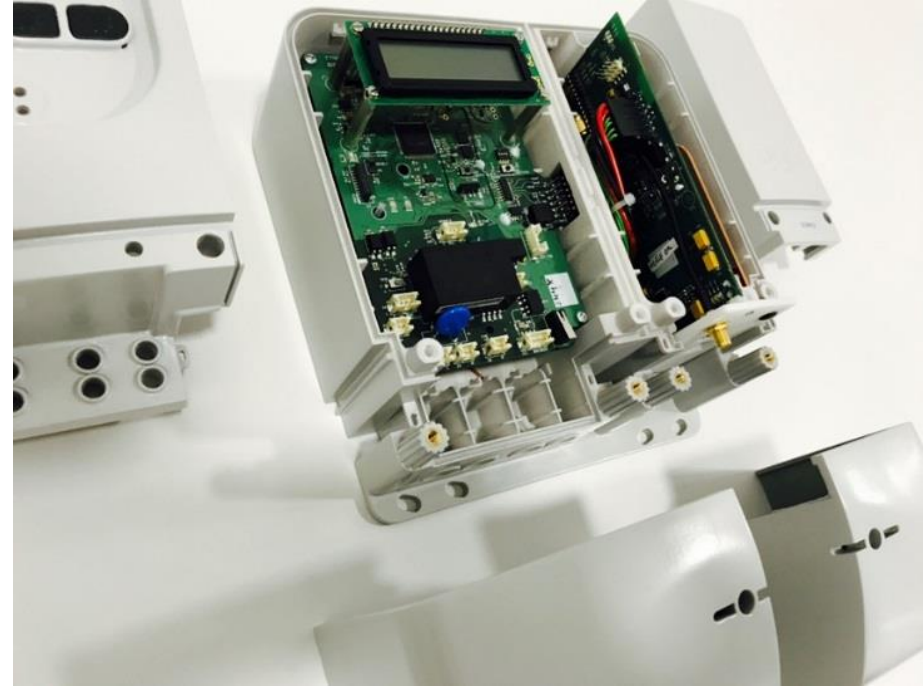

Fig. 2.

SLAM: Next generation smart meters by NOBEL GRID

On the other hand, in the case of retailers, the SLAM services facilitate the tariffication and billing of their customers, supporting personal data security and user privacy.

Regarding final consumers and prosumers, the SLAM provides them with more protection and empowerment, facilitating the monitoring and management of their consumption, production and energy efficiency, making possible automatic demand response schemes. For instance, by means of the SLAM, the consumer is able to communicate and interact remotely with the intelligent devices in order to adapt their energy use habits and optimize energy consumption. These devices can be controlled remotely via an App provided also by NOBEL GRID project.

It is in this last context where the SLAM offers new opportunities to energy communities and cooperatives, by providing information of the aggregated flexibility to the grid. This aggregated information makes possible collective demand response schemas and flexibility strategies in order to make use of the energy in an optimal way, reducing electricity billing and carbon emissions.

Direct control of energy data by energy communities is the first and necessary step to boost marketing of flexibility driven by end-users. The SLAM goes beyond the traditional functionalities of digital smart meters -i.e. power control and billing - and provides the end-user with an intelligent device, similar to a network analyser - capable of not only retrieve more granular information to enrich the observability of the distribution network, but also of providing the prosumer with an open platform for the deployment of added value services. In this sense, not only energy communities, but also Energy Service Companies (ESCOs), retailers and aggregators are then able to offer specific strategies to maximize the benefit of the end-user building on-top od such new services, which are only feasible thanks to the direct access and control of energy data. 
Moreover, this new generation of smart meter, by increasing the controllability of the information flow by end-users, foster richer privacy and data protection schemas, allowing the data-owner to know at any time who has been granted with data access rights and with which granularity and frequency the data is being exposed to the different service providers.

The SLAM has been deployed and validated in 5 pilot sites around Europe in the context of NOBEL GRID:

- CARBON COOP: Energy cooperative aggregator with members in Manchester and Lancaster (United Kingdom).

- ECOPOWER: Renewable energy cooperative based in Flanders (Belgium)

- ECA (Electricity Cooperative of Alginet - Spain): Cooperative of energy distribution and commercialization.

- ASM Terni: Public distribution system operator based in Terni (Italy).

- Meltemi (Greece): Eco-village with its own micro grid close to Athens.

By means of the deployment of SLAM in these pilot sites, the project partners have been able to participate actively in the energy market and in demand response schemes; demonstrating and validating new business models defined and assessed within NOBEL GRID under real conditions. Through these new business models, the SLAM promotes empowerment and protection of the final consumers and collective flexibility services. Most of the project pilot sites are energy cooperative, therefore, the benefits provided by the SLAM are shared in a democratic way among all the members of the cooperative.

Furthermore, the SLAM has been already identified as one of the main solutions in order to improve smart grid in other scenarios beyond NOBEL GRID. In this context, the SLAM is being also deployed in other research projects such as the H2020 projects WiseGRID and MatchUp.

\section{Market your flexibility: wise cooperatives}

The control and management of their own energy metering data, can allow energy cooperatives and aggregations of prosumers to improve their position in the overwhelming electricity sector. This is the first step to become a Wise Cooperative and take maximum advantage of the collaboration between different types of prosumers.

In most cases, those cooperatives are willing to increase RES in the energy mix by means of higher penetration of DER and the aim of achieving the self-management of their energy resources. These new smarter cooperatives will be one of the leading vectors in the energy transition and it is mandatory to provide them user-friendly and powerful tools.

Towards these objectives, the WiseGRID project [2] has developed a solution specifically focused on satisfying the needs of these kind of energy communities. WiseCOOP is a tool that help cooperatives to optimize the use of their green DER and monetize their energy flexibility and zero-emission production.

In the WiseGRID project, the required metering data for correctly running the WiseCOOP is coming from the SLAMs already deployed in 3 of the Pilot Sites of NOBEL GRID (Terni, Meltemi and Flanders). The characteristics and functionalities of the SLAM perfectly adapts the requirements of the $\mathrm{W}$ iseCOOP tool and thanks to the Linux based module of this smart meter, both products are able to evolve in parallel.

The main goal of WiseCOOP is helping consumers and prosumers to work together in order to achieve better energy deals. In the particular scenario of increasing share of distributed renewable energy resources, this goal can be achieved by providing the following advanced functionalities for cooperatives:

- Supporting the operation of communities of prosumers that invest in renewable energy sources aiming to be as much self-sufficient as possible.

- Providing clear information to members to raise awareness on efficient energy usage and environmental practices.

- Implicit price-based Demand Response (DR) participation. This is achieved by modulating the overall demand of the group to achieve a common objective (as, for instance, maximize usage of renewable energy sources produced within the group. Taking into account the aggregated optimized flexibility of its members, the cooperative, through the WiseCOOP tool is able to participate in DR campaigns and flexibility markets.

- Explicit DR campaigns participation. The members of the cooperative are able to answer the requests of a Grid Operator in order to solve different issues in the electricity network.

The functionalities above mentioned are available thanks to the specific and accurate internal architecture and module design of the tool. The main processes which internally run $\mathrm{W}$ iseCOOP are:

- Tariff comparison: by offering members a tool for comparing their particular consumption with different available tariffs, those will have access to very valuable information to reduce their energy bills.

- Member profiling: clusters of consumers and prosumers with common energy usage patterns may be identified, allowing the aggregator to negotiate special terms (as for instance energy tariffs) particularly beneficial for those groups.

- Demand and production forecasting: by allowing the retailer to forecast the demand of its customers then, optimized purchase of energy at the wholesale market is enabled. 
- Flexibility estimator and DR campaign scheduler. Taking into account the demand and production forecast and the comfort preferences of the user, this module calculates the flexibility of each member of the cooperative and allows the participation in DR schemas.

- Connection with external actors. The tool communicates with external actors of the energy framework such as a Grid Operators and energy markets. Also can get data from external devices like the SLAM or weather stations.

There are several devices which are able to provide flexibility to the WiseCOOP and the WiseGRID project will take all of them into account in a holistic approach. Thus, the cooperatives of consumers and prosumers can take advantage of their different energy consuming assets whatever their nature or their size. In the WiseGRID context, the flexibility sources are mainly batteries, EVs (Electric Vehicles), micro CHP (micro cogeneration), solar panels, HVACs and other shiftable consuming devices.

Each kind of flexibility source will be managed by a different tool of the WiseGRID framework:

- WG STaaS/VPP manages and controls the batteries at consumer and MV level.

- WiseEVP handles the flexibility that a single EV or an EV fleet can provide to the system.

- WiseCORP manages and controls the HVACs, micro CHPs, solar panels and other shiftable consuming devices of a building or group of buldings.

To better explain the interaction between WiseCOOP and the other tools, it is necessary to differentiate the role of WiseCOOP in the explicit DR context and in the implicit DR context.

In an explicit DR situation, the DSO (through other tool called WG Cockpit) sends to the different aggregators a DR request in order to solve a problem in the MV or LV grid. WiseCOOP answers this DSO request with an offer (in terms of price and flexibility available). To make this response, WiseCOOP has to previously collect data from WiseCORP (for example building flexibility information). As a remark, WG STaaS/VPP and WiseEVP can also send offers to the DSO.

In case the DSO accepts the offer of the aggregator using WiseCOOP, this product will send the required commands to WiseCORP in order to properly answer WG Cockpit needs.

In an implicit DR situation, WiseCOOP studies and processes the demand and production forecast of WiseCORP, WG StaaS/VPP and WiseEVP (in a day ahead time frame) in order to send them post-day ahead price signals that will help to balance the retailer's portfolio.

As explained here above, this aggregated flexibility can be offered to system operators aiming to alleviate congestion and stability problems. It is in this context where new opportunities for local and regional collaboration may arise.
Aggregators and energy communities will act as flexibility service providers to both DSO and TSOs. Thus it is possible to find Win-Win situations in which both Grid Operators and cooperatives of prosumers can get benefits of their mutual collaboration.

\section{Local and regional opportunities: DSO-TSO collaboration}

With a higher real-time resolution metering, and the collaboration of aggregation parties, it will be possible to evaluate the direct impact on electricity market and RES penetration limits, providing the grid with power system dynamics and awareness capabilities in case of operation of the power system close to the stability limits. In this respect, Wide Area Monitoring and Awareness Systems (WAMAS) providing event recording, real-time monitoring, phasorassisted state estimation, real-time congestion management, and recognition of instabilities, are critical to efficiently handle the new opportunities of collaboration DSO-TSO, at local and even at regional level.

One aspect that needs to be further developed is the measurement of the real contribution of each ancillary services participant - i.e. aggregator, by measuring the executed service and comparing it with the order, thus being able to define appropriate Quality of Service (QoS). This process of cross-checking/verification can only be done for the aggregation of many small contributors (acting e.g. as a Virtual Power Plant, VPP, delivering a certain ancillary service), where the contributor's trustfulness is otherwise difficult to assess and prequalification is difficult to be made for so many small resources.

This is the approach that is being followed by the CROSSBOW project [3]. The one to five minute load profiles of energy meters will be considered as legal and metrology-based primary source for such measurements and assessments. This has the intention to improve the fairness and transparency of the service paid, thus improving fairness in settlement of balancing services, for both aggregated services and ancillary services [4]. This will require advanced load profiling at distribution level and flexibility at TSO/DSO interconnection points in order to assess how Demand Side Management (DSM) can contribute to ancillary services for system operators. CROSSBOW will consider aggregated resources from storage, generation and demand response, as assets for participating in ancillary services markets for local and regional markets.

The project is specifically tackling the coordination of DER distributed along different countries in South Eastern Europe (SEE). CROSSBOW proposes the shared use of resources to foster cross-border management of variable renewable energies and storage units, enabling a higher penetration of clean energies whilst reducing network operational costs and improving economic benefits of RES and storage units.

This cross-border approach, open new opportunities for the collaboration between TSOs, but also between TSO-DSO at 
both local and transnational level. The project will provide coordination tools for the management of RES, storage and DR. It will also provide an Ancillary Market toolset to support different flexibility providers to trade with system operators. It is in this context where wise cooperatives - or aggregation of prosumers represented by a professional third party - would be able to maximize their assets value, participating in the market not only as consumers or producers -i.e. trading energy- but also as flexibility providers.
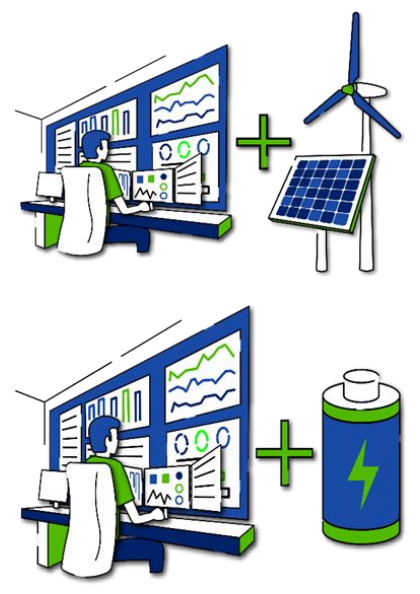

Fig. 3. CROSSBOW coordintation centers (RES and Storage)

In CROSSBOW, an innovative business model with underlying ICT technology for Cooperative Ownership of Flexibility Assets (CFP) will be specified, developed, demonstrated and validated. Although Flexibility Assets, like Demand Response systems and Virtual Power Plants, are becoming common providers of balancing services in some European markets (Austria, France, Slovenia, UK, etc.) they are normally owned and operated only by electricity retailers or independent aggregators which business objectives might not necessarily be aligned with their providers of flexibility (consumers, prosumers, distributed generators, RES, storage etc.). The cooperative ownership concept will be aligned with existing regulation and general enough to support existing flexibility assets, like VPPs and DR systems. Furthermore, it will by design enable multinational ownership of flexibility assets, which will increase the business opportunities and decrease some of the today's technology uptake barriers.

Especial attention will be paid to the collaborative ownership of storage assets - distributed or not. A set of distributed storage units (in the same country or nor) will be able to be used by system operators in order to contribute to the quality of electricity supply (e.g., voltage level control through Q management) and to keep the grid balanced through strategies of distributed $\mathrm{P}$ control whilst reducing operation costs for the entire energy system. This ancillary service could be covered by aggregation of storage units owned by different prosumers. The objective will be to use the distributed storage units to operate the grid more efficiently and at lower costs while maintaining the grid stability. This will be achieved in CROSSBOW through the development of advanced algorithms that would allow the optimal management and coordination of distributed, cross-border storage units participating in a common coordination center in order to provide multiple ancilliary services. The implementation of such algorithms will ensure the optimal operation of smart grids and will coordinate the required actions in order to utilize and maximize the benefits from distributed, cross-border energy storage units. It is therefore the vision to develop techno-economic frameworks and algorithms for advanced coordinated use of distributed storage units for improvement of cross-border power transfers and associated issues (e.g., voltage and frequency regulation) at national and regional level.

For Wise Cooperative to be able to efficiently participate in the common storage coordination center and ancillary market foreseen by CROSSBOW, they will have to group their storage as sets as a Virtual Storage Plant.

A Virtual Storage Plant (VSP) is an aggregation of energy storage resources that can: i) be geographically spread across different locations; ii) be treated as a single large storage resource from the system operator's perspective; iii) use any combination of energy storage technologies, (small, e.g., batteries, super capacitors, flywheel, superconducting magnetic energy storage, and large e.g., compressed air, hydro) to provide grid support in existing and future transmission networks at regional and global level. CROSSBOW will propose a framework for different stakeholders to promote and adopt the use of VSP. System Operators will be offered specific services to use VSP as primary and secondary reserve, congestion management and transmission and distribution deferral; whilst energy producers and retailers will be able to use VSP to mitigate imbalance, store energy surplus and modulate market offers considering not only the production, but also the storage capability for steady state and dynamic support of the network and provision of required quality of supply.

Some of the existing or envisaged operational issues identified by the system operators participating in CROSSBOW could be mitigated with the use of future VSP assets. And due to the distributed nature of a VSP, the participation of wise cooperatives, though the new business model provided by the CFP, is the best way to promote the adoption of VSPs as local flexibility enablers.

Therefore, an efficient cooperation between TSOs-DSOs and end-users can solve is sues due to increased RES penetration, such as voltage limits violation and congestions, by utilizing VSP units. Voltage limits violations occur mainly due to excessive reverse power flows from the distribution network to the transmission system while congestions may occur both due to high demand and increased RES production. TSO and DSO can cooperate in order to enable the adequate distribution network flexibility coming from VSP owned by empowered energy communities to solve voltage violations/congestions. 


\section{Acknowledgements}

This paper has received funding from the European Union's Horizon 2020 Research and Innovation Programme under Grant Agreement N 773430 (CROSSBOW); N 646184 (NOBELGRID); N 731205 (WISEGRID).
[1] www.nobelgrid.eu. On-line 01-09-2018.

[2] www.wisegrid.eu. On-line 01-09-2018.

[3] www.crossbowproject.eu. On-line 01-09-2018.

[4] C. C. L. T. L. A. L. P. Mihai Sănduleac, National and inter-TSO Balancing and Ancillary Services Markets Within a Pyramid of Energy Services, Belgrade, Serbia: IET MEDPOWER 2016, 6-9 Nov em ber 2016.

\section{References}

